\section{PTU-032 MANAGEMENT OF AUTOIMMUNE HEPATITIS A UK-WIDE SURVEY}

doi:10.1136/gutjnl-2012-302514c.32

H Dhaliwal, ${ }^{*}$ C Facey, D Gleeson. Liver Unit, Sheffield Teaching Hospitals NHS Trust, Sheffield, UK

Introduction Most hepatologists and many gastroenterologists manage patients with autoimmune hepatitis (AIH). There are no data regarding management of $\mathrm{AIH}$ in the UK as a whole. We aimed to conduct a survey of UK clinicians' practice in regard to managing AIH. Methods An anonymised online questionnaire was e-mailed to 952 UK gastroenterology and hepatology consultant physicians, identified via the Directory of Gastroenterology. ${ }^{1}$ Responses were collected via surveymonkey, an online questionnaire tool. We included only responders who indicated that they managed patients with liver disease. We defined responders as hepatologists if liver disease made up $>70 \%$ of their workload.

Results $228(24 \%)$ responded, 222 of whom managed patients with liver disease (38 (17\%) hepatologists and 184 (83\%) gastroenterologists). $26 \%$ managed $\leq 5$ patients with $\mathrm{AIH}, 25 \% 6-10,30 \%$ $11-25,10 \% 26-50$ and $8 \%>50$ patients. Half the responders indicated that all gastroenterologists in their hospital managed AIH; the remainder indicated that $\mathrm{AIH}$ was managed by hepatologists or gastroenterologists with a "liver" interest. Diagnostic liver biopsy (in absence of serious contraindication) was performed always by $62 \%$, usually by $27 \%$ and only if the diagnosis was in doubt by $11 \%$. Hepatologists were more likely than gastroenterologists to perform diagnostic liver biopsy always ( $82 \%$ vs $58 \%$; $=0.02$ ). As routine initial therapy, $80 \%$ of respondents used prednisolone+azathioprine (PRED+AZA) combined, 12\% used PRED alone and 3\% used budesonide + AZA. $65 \%$ continued PRED only until serum ALT normalised or for up to 6 months. $20 \%$ did so for $>12$ months and $15 \%$ did so until confirmation of histological remission. Gastroenterologists were more likely than hepatologists to continue PRED only until ALT normalised or for up to 6 months ( 68 vs $50 \%$ ) and were less likely to do so until confirmation of histological remission (12 vs $29 \% ; p=0.02) .17 \%$ of respondents repeated liver biopsy routinely to confirm histological remission, $67 \%$ did so in selected cases and $16 \%$ never did. $63 \%$ routinely used maintenance therapy and 30\% did so only after first relapse. Preferred maintenance therapy was AZA monotherapy in $74 \%$ and AZA+PRED in $21 \% .34 \%$ of respondents continued maintenance therapy indefinitely, $59 \%$ attempted withdrawal (6\% after $2-5$ years; $50 \%$ after $5-10$ years and $3 \%$ after $10-20$ years) and $7 \%$ had a personalised approach. There were no differences between hepatologists and gastroenterologists regarding repeat liver biopsy policy or maintenance therapy.

Conclusion In the UK, there is much variation in the approach to managing AIH, specifically in regard to: role of liver biopsy, initial therapy (particularly duration of steroids) and use of maintenance therapy.

Competing interests None declared.

\section{REFERENCE}

1. Directory of Gastroenterology 2011. 5th edn. Mediahuset.

\section{PTU-033 EVALUATION OF BONE HEALTH IN CORTICOSTEROID- TREATED AUTOIMMUNE HEPATITIS}

\section{doi:10.1136/gutjnl-2012-302514c.33}

${ }^{1} \mathrm{H}$ Dhaliwal, ${ }^{*} \mathrm{~N}$ Peel, ${ }^{1} \mathrm{D}$ Gleeson, M Karajeh. ' Liver Unit, Sheffield Teaching Hospitals NHS Trust, Sheffield, UK; ${ }^{2}$ Metabolic Bone Unit, Sheffield Teaching Hospitals NHS Trust, Sheffield, UK

Introduction Corticosteroid use, cirrhosis and inflammatory disease are risk factors for osteoporosis. However the occurrence of osteo- porosis in Autoimmune Hepatitis (AIH) has been little studied. Our aims were to evaluate retrospectively the effects of the disease and its treatment on bone mineral density (BMD) in AIH and whether the use of bisphosphonates prevents bone loss.

Methods 108 patients, median (range) age 60 (18-80) with AIH for 7 (1-416) months were included. BMD was measured using dualenergy x-ray absorptiometry (DXA) at the hip and/or lumbar spine. Results are compared to manufacturer's reference population and expressed as T- and Z-scores. To assess the effect of intervention, we studied a subgroup of 37 prednisolone-treated patients who had a second DXA, with the first performed at $3(1-6)$ months and the second at 28 (15-67) months from diagnosis. Patients were prescribed a bisphosphonate after the $1^{\text {st }}$ DXA if clinically indicated. Results $20 \%$ of patients had osteoporosis and $30 \%$ had osteopenia. The mean \pm SD Z-score at the hip was $0.1 \pm 1$ and at the spine $0.1 \pm 1.5$, which was not significantly different from that of the reference population ( $\mathrm{p}=0.4$ for both). Compared to those with normal $\mathrm{BMD}$, patients with osteoporosis had higher fibrosis stage at diagnosis (median 3 vs $5, p=0.006$ ). On multivariate analysis, hip Z-score showed independent associations: negative with log cumulative prednisolone dose $(\mathrm{r}=-0.25, \mathrm{p}=0.02)$ and positive with body weight $(\mathrm{r}=0.4, \mathrm{p} \leq 0.001)$, but there was no association with disease duration. 19 patients had fragility fractures. Patients with a fracture had lower hip and spine T-scores compared to patients without (hip T-score -1.8 vs $-0.6, p=0.006$, spine T-score -2.2 vs $-1.0, p=0.015)$. In the paired DXA analysis, BMD in hip and spine increased in patients $(n=18)$ commenced on a bisphosphonate after the $1^{\text {st }}$ DXA compared to those who were not (\% change in BMD/ year at hip +0.3 vs $-1.7, p=0.002$ and at spine +2.1 vs -2.0 , $\mathrm{p}=0.002$ ), despite the two groups receiving similar current and cumulative prednisolone doses.

Conclusion Mean BMD in treated AIH is not lower than the expected level for age. Prednisolone dose-related bone loss occurs but can be prevented with appropriate use of bisphosphonates.

Competing interests None declared.

\section{PTU-034 HISTOLOGICAL REMISSION IN AUTOIMMUNE HEPATITIS CAN IT BE PREDICTED?}

doi:10.1136/gutjnl-2012-302514c.34

${ }^{1} \mathrm{H}$ Dhaliwal, ${ }^{*} \mathrm{~B}$ Hoeroldt, ${ }^{3} \mathrm{~A}$ Dube, ${ }^{1} \mathrm{E}$ McFarlane, ${ }^{1} \mathrm{M}$ Karajeh, ${ }^{1} \mathrm{D}$ Gleeson. ${ }^{1}$ Liver Unit, Sheffield Teaching Hospitals NHS Trust, Sheffield, UK; ${ }^{2}$ Department of Gastroenterology, Rotherham General Hospital, Rotherham, UK; ${ }^{3}$ Pathology, Sheffield Teaching Hospitals NHS Trust, Sheffield, UK

Introduction A recent report ${ }^{1}$ suggested that initial treatment of Autoimmune Hepatitis (AIH) with high-dose prednisolone results in more rapid normalisation of serum ALT and (by implication), improved outcome. However, we have reported ${ }^{2}$ that persisting histological activity in AIH (Ishak necroinflammatory score (NIS) $>3$ ), despite serum ALT normalisation, is associated with fibrosis progression and increased mortality. Here, we aimed to identify, retrospectively, disease or treatment related factors associated with attaining histological remission.

Methods We studied 111 patients with AIH (93 female, median (range) age at diagnosis 57 (14-77) years treated with prednisolone plus azathioprine/mycophenolate, who had attained normalisation of serum ALT and had available follow-up biopsy.

Results Starting daily doses of prednisolone and of azathioprine were $30(10-60)$ and $50(25-150) \mathrm{mg}$ respectively. Time taken to achieve normal serum ALT after starting treatment was 8 (0-144) weeks and showed no correlation with any of: (a) gender, age, serum ALT, or globulin at presentation (b) NIS or fibrosis grade at diagnostic biopsy, (c) starting dose of prednisolone or azathioprine. Biopsy was repeated 26 (12-90) months after starting 
treatment. 72 patients (65\%) attained histological remission. Comparing these with the 37 patients not in histological remission, there were no significant differences in age, gender, presenting serum ALT, or in NIS or fibrosis stage at diagnostic biopsy. Neither were there differences in either (a) starting or cumulative dose of prednisolone or azathioprine (either absolute or corrected for body weight) or (b) time to achieve normal serum ALT. Only serum globulin at presentation was lower in those who achieved histological remission (42 vs $49 \mathrm{~g} / \mathrm{l}, \mathrm{p}=0.01$ ). On multivariate analysis, NIS at follow-up biopsy was independently associated with serum ALT at time of biopsy $(p \leq 0.001)$ but with none of above baseline, treatment or early response -related variables.

Conclusion We could not identify baseline or treatment -related variables associated with (and thus, potentially predictive of) histological remission. Specifically, we could not establish that histological remission was related to either time to serum ALT normalisation or to starting or cumulative dose of prednisolone.

Competing interests None declared.

\section{REFERENCES}

1. Schramm C, et al. Hepatology 2010;52:2247-82.

2. Hoeroldt BS, et al. Gut 2009;58:A18.

\section{PTU-035 AZATHIOPRINE METABOLITE CONCENTRATIONS IN AUTOIMMUNE HEPATITIS: RELATIONSHIP TO DISEASE REMISSION}

doi:10.1136/gutjnl-2012-302514c.35

${ }^{1,2} \mathrm{H}$ Dhaliwal, ${ }^{*}{ }^{2} \mathrm{E}$ Thornhill, ${ }^{2} \mathrm{R}$ Anderson, ${ }^{1} \mathrm{E}$ McFarlane, ${ }^{1} \mathrm{D}$ Gleeson, ${ }^{2} \mathrm{~L}$ Lennard. ${ }^{1}$ Liver Unit, Sheffield Teaching Hospitals NHS Trust, Sheffield, UK; ${ }^{2}$ Department of Human Metabolism, University of Sheffield, Sheffield, UK

Introduction Azathioprine (AZA) is used to maintain remission in AIH but is sometimes ineffective. AZA is a pro-drug and formation of active thioguanine nucleotide (TGN) metabolites varies widely. Competing with TGN formation is drug methylation, catalysed by thiopurine methyltransferase (TPMT). The methylated drug is inactive, but the methyl-mercaptopurine nucleotide metabolites (MeMPs) may not be. Previous studies, based mainly on single measurements, have not shown a relationship between TGN levels and clinical efficacy. We assessed the relationship between mean of several metabolite levels (TGNs and MeMPs), and therapeutic response in $\mathrm{AIH}$ patients prescribed a constant dose of AZA for remission maintenance.

Methods Erythrocyte TGNs and MeMPs were measured on serial blood samples by established techniques over 2 years. Average TGNs (avTGNs) and MeMPs (avMeMPs) concentrations for each patient at a constant AZA dose were analysed. Therapeutic response was defined as maintenance of remission on a given dose of AZA. Relapse was defined by the IAIHG criteria (ALT $>2 \times$ ULN or symptoms + ALT $>$ ULN).

Results 349 samples from 68 patients (median 5, range 2-9, per patient) were analysed. Median avTGN concentration was $220 \mathrm{pmol} / 8 \times 10^{8} \mathrm{RBC}$ (range 66-888) at a median dose of $1.9 \mathrm{mg} /$ $\mathrm{kg} /$ day. AZA dose $(\mathrm{mg} / \mathrm{kg} /$ day) correlated positively with avMeMPs $\left(r_{s}=0.38, p=0.001\right)$ but not with avTGN $\left(r_{s}=-0.06, p=0.6\right)$. Patients in whom remission was maintained compared to those who relapsed had higher avTGN concentrations (Abstract PTU-035 table 1) but avMeMPs concentrations were not significantly different. Mean ALT (both over the metabolite monitoring period and over the time on the constant AZA dose) correlated negatively with avTGN $\left(r_{s}=-0.322, p=0.007\right.$ and $\left.r_{s}=-0.342, p=0.004\right)$ but not with avMeMPs. Patients who maintained a normal ALT had higher avTGN concentration compared to those who did not (median 237 vs $177 \mathrm{pmol} / 8 \times 10^{8} \mathrm{RBC}$ ).
Conclusion In patients with AIH maintained on AZA, lower average TGN concentrations are related to development of relapse and to higher ALT.

\section{Abstract PTU-035 Table 1}

\begin{tabular}{|c|c|c|c|}
\hline & $\begin{array}{l}\text { Group 1: Remission } \\
\text { maintained, median } \\
\text { (range) } \mathbf{n}=\mathbf{5 3}\end{array}$ & $\begin{array}{l}\text { Group 2: Relapse while } \\
\text { on treatment, median } \\
\text { (range) } n=15\end{array}$ & p Value \\
\hline AZA dose, $\mathrm{mg} / \mathrm{kg} /$ day & $1.8(0.4-2.7)$ & $2.0(0.9-3.2)$ & 0.2 \\
\hline Time of Dose, months & $41(1-208)$ & $11(2-216)$ & 0.008 \\
\hline avTGN pmol/8×108 RBC & $236(66-888)$ & $177(67-400)$ & 0.04 \\
\hline avMeMP pmol/8×108 RBC & $1428(189-23798)$ & 1041 (175-18 879) & 0.4 \\
\hline
\end{tabular}

Competing interests None declared.

\section{PTU-036 MYCOPHENOLATE MOFETIL IN PATIENTS WITH AUTOIMMUNE HEPATITIS AND AZATHIOPRINE INTOLERANCE: HISTOLOGICAL RESPONSE COMPARED TO THAT IN AZATHIOPRINE-TREATED PATIENTS}

doi:10.1136/gutjnl-2012-302514c.36

${ }^{1} \mathrm{H}$ Dhaliwal, ${ }^{*} \mathrm{E}$ McFarlane, ${ }^{2} \mathrm{~A}$ K Dube, ${ }^{1} \mathrm{D}$ Gleeson, ${ }^{1} \mathrm{M}$ Karajeh. ${ }^{1}$ Liver Unit, Sheffield Teaching Hospitals NHS Trust, Sheffield, UK; ${ }^{2}$ Pathology, Sheffield Teaching Hospitals NHS Trust, Sheffield, UK

Introduction Prednisolone (PRED) in combination with azathioprine (AZA) is commonly used to induce remission in autoimmune hepatitis (AIH). Up to $20 \%$ of patients are intolerant of AZA and mycophenolate mofetil (MMF) is often used as an alternative. However there are few data on its ability to induce histological remission and prevent relapse. Therefore, we aimed to evaluate the efficacy of MMF, compared to AZA, in inducing and maintaining remission

Methods 58 patients with AIH presenting since 2003 were retrospectively studied. All initially received PRED+AZA. Those who developed intolerance to AZA ( $\mathrm{n}=13$, after 1-6weeks) were switched to MMF (1 (1-2) g/day). Follow-up liver biopsy, obtained after median $25(12-41)$ months was assessed in 11 of these patients (MMF group) and 44 patients in whom AZA was continued (AZA group).

Results The two groups were similar with regards to (a) serum ALT at $0,3,6,12,24$ and 36 months, and (b) Ishak necroinflammatory grade at baseline (11 vs 10 in AZA and MMF group respectively, $\mathrm{p}=0.6$ ) and follow-up (4 vs 3, $\mathrm{p}=0.9$ ) biopsy. $48 \%$ of the AZA group and $64 \%$ of the MMF group achieved histological remission $(p=0.3)$. Fibrosis regressed in both groups but regression was greater in the MMF group (Ishak stage 3.2 to $1.7, \mathrm{p}=0.002$ ) compared to the AZA group ( 3.3 to $2.8, p=0.02$ ). When attempted, PRED was successfully withdrawn in $7 / 10$ patients in the MMF group, and in $32 / 37$ patients in the AZA group ( $p=0.3)$. After PRED withdrawal, 4/7 patients in the MMF group and 11/32 patients in the AZA group relapsed ( $p=0.4)$. Up to the end of follow-up (71 months for AZA vs 65 months for MMF, $p=0.6$ ) PRED requirement (median 0 vs $2.5 \mathrm{mg}, \mathrm{p}=0.8$ ) and relapse rate (median 0 vs $0, \mathrm{p}=0.5$ ) were similar in the two groups.

Conclusion MMF is as effective as AZA, at inducing and maintaining biochemical and histological remission in $\mathrm{AIH}$. It may lead to more regression of fibrosis but this requires further evaluation.

Competing interests None declared. 